\title{
Study of a Micro Thermal Environment of a Personal Air-Conditioning
}

\author{
Nguyen Anh Tuan and K. David Huang
}

\begin{abstract}
In a highly developed living, people are always looking for a comfortable indoor environment with minimum energy use. Regional air conditioning mechanism (RACM) can create a personal thermal comfort control in a workroom which can contribute to save air-conditioning energy. In this study, we analyze the airflow circulation cell of the RACM with varied inlet port opening and inlet port position dimensions using the computational fluid dynamics (CFD) technique. We created a RACM, two workstations, lightings, and a cabinet in a 3-dimensional room. The fluid was assumed to be Newtonian, unsteady, and incompressible. A Bossinesq approximation was determined in order to consider the buoyancy effect. We examined the effects of the inlet port opening and inlet port position on airflow circulation establishing process. Air temperatures along the various midline of the occupied zone were predicted and compared for a range of inlet port opening and inlet port position by using non-dimensional form. We also showed the occupied zone temperature at various planes in the workroom. Results will indicate the suitable inlet port opening and inlet port position for maintaining individual satisfied occupants' requirements and improving energy saving potential.
\end{abstract}

Index Terms-Environmental thermal comfort, personal air-conditioning, computational fluid dynamics (CFD), energy-saving.

\section{INTRODUCTION}

The cooling costs will be increased when the air-conditioning system is turned on in the room. Home energy saving tips point out that, under setting the thermostat to $25.5 \mathrm{oC}$, the cooling costs will be saved of $10-20 \%$, and 5 - $12 \%$ in case of 29.4 oC when you leave your home for more than four hours [1]. The total electric energy consumption is reduced by about $30 \%$ to $40 \%$ in room without air conditioning [2]. Since the increasing demand for building services, growing in population, together with the rise in time spent inside buildings, assure the upward trend in energy demand will continue in future. If cool air could be directed to desired zone (s) in a room, the cooling energy consumption will decrease, so contributing to energy issue thrift. There were some studies concerning these problems with theory of personal air-conditioning, so far.

Zeng et al. [3] showed personalized ventilation systems (PVS), whose design allowed each occupant to control

Manuscript received July 11, 2013; revised September 13, 2013.

Nguyen Anh Tuan is with the Department of International Cooperation, Ministry of Science and Technology, Hanoi 10000, Vietnam, (email: tuana102000@yahoo.com or anhtuan@most.gov.vn)

K. David Huang is with Institute of Mechanical and Electrical Engineering, National Taipei University of Technology, Taipei 10608 , Taiwan, R.O.C (email: kdavidh@ntut.edu.tw). his/her air flow direction, temperature and select his/her thermal comfort demands. In their studies, the perceived air quality of the PVS is superior to the conventional mixing ventilation system under the same amount of supply air.

Melikov and Fanger [4]-[6] have systematic reviews on the performance of personalized ventilation (PV) and on human response to it. $\mathrm{PV}$, in comparison with traditional ventilation, has two important advantages: (i) it has potential for improving the inhaled air quality, and (ii) each occupant is delegated the authority to optimize as well as control the temperature, the flow rate, and the direction of supplied air according to their own preferences and to thermal comfort requirements.

In 2005, Huang et al. [7] adopted airflow management technology to improve regional airflow temperature distributions in an automobile to counteract the greenhouse effect by using computational fluid dynamics (CFD) method. An auxiliary power supply system that automatically powers the "greenhouse" ventilation system using solar-energy was shown in that study. The auxiliary solar-power supply can save energy and reduced the green effect of sunlight, while creating a comfort traveling environment. In 2006, Huang et al. [8] presented air-conditioning system of an intelligent vehicle-cabin to satisfy with respect to occupant's unique temperature demands, which can control regional airflow circulation in certain areas. The experimental measurement was conducted to test the influences on regional air-conditioning system as a result of introducing without manikin, with manikin, and a real person in the cabin. In recent published designs, the author [9]-[12] also investigated CFD simulations and experimental works in workroom with the theory of regional air-conditioning mechanism (RACM). However, most of previous studies on airflow circulation cell in empty workrooms have been dealt with limited range of physical and geometrical parameters. This investigation considered airflow circulation cell establishing process in a workroom with two workstations in the room for a various inlet port opening and outlet port position. The inlet port parameters are non-dimensionalized with RACM wall so as to the generalizability of results from this paper.

\section{WORKING ROOM MODEL}

A 3-dimensional RACM with height $(H)$ of $2 \mathrm{~m}$ and diameter $(D)$ of $0.075 \mathrm{~m}$ is modelled inside a room with internal dimensions of $10 m \times 5 m \times 3.2 m$ (ceiling height) which is considered the atmosphere as shown in Fig. 1. Room has two workstations, one located in middle room, and the other was at corner room. Fig. 2 shows the computational 
domain modelled using Gambit software [13].

A non-dimensional variable $(W)$ is considered, it is defined as the ratio between the inlet port height $(w)$ to the height of $\operatorname{RACM}(H)$. Another non-dimensional variable $(L)$ is defined as the ratio between the inlet port position $(l)$ and the height of RACM $(H)$.

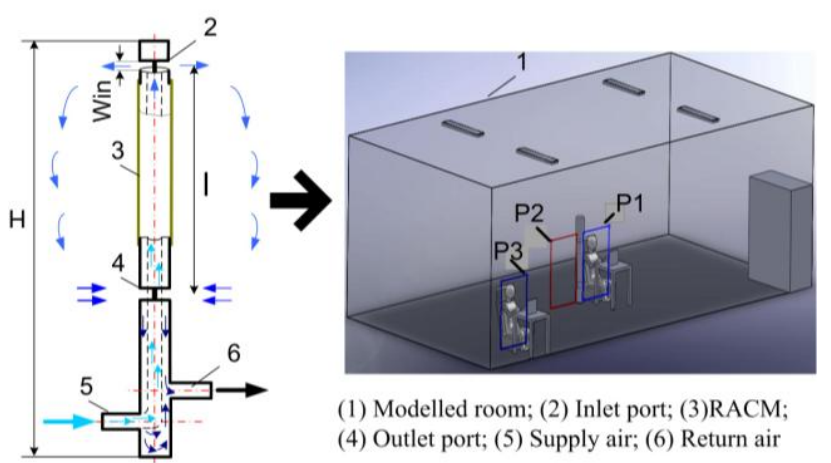

Fig. 1. Model of the RACM in the modeled room

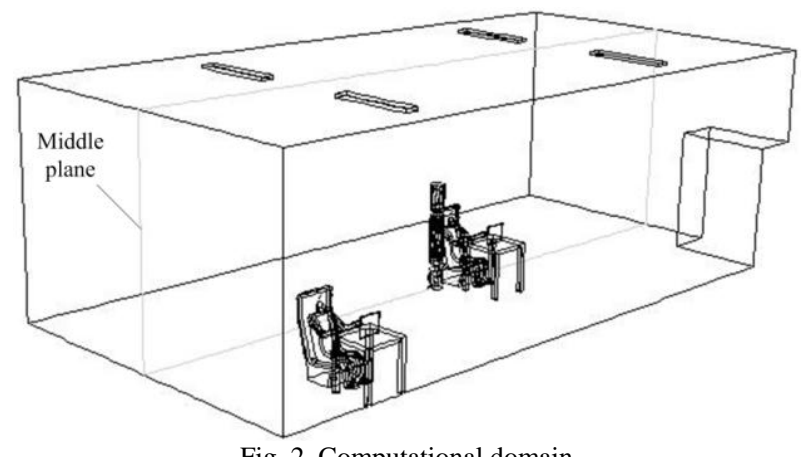

Fig. 2. Computational domain

TABLE I: ANALYSIS CASE DETAILS

\begin{tabular}{lcc}
\hline \hline & Equations & Values \\
\hline $\begin{array}{l}\text { Changing the inlet port } \\
\text { opening of the }\end{array}$ & $W=\frac{\mathrm{w}_{\text {in }}}{H}$ & $0.0117,0.0235,0.0352$, \\
$\begin{array}{l}\text { RACM- a } \\
\text { non-dimensional } \\
\text { number }\end{array}$ & & $0.0470,0.0588,0.0705$ \\
$\begin{array}{l}\text { Inlet port position - a } \\
\text { non-dimensional } \\
\text { number }\end{array}$ & $L=\frac{l}{H}$ & $0.176,0.294,0.470$, \\
\hline \hline
\end{tabular}

\section{METHOD}

The fluid within the room is considered to be an unsteady incompressible, the flow within the enclosure is assumed to be turbulent, Boussinesq approximation which was valid to consider the gravity and buoyancy effects.

To nondimensionalize these governing equations as shown in equations (1), (2), (3), (4), (5), and (6) divide all lengths by the height of the RACM, H and all velocity by inlet fluid velocity, ui. As for temperature the two values (Tin and Tw) are used. Make the pressure nondimensional by dividing by $\rho u_{i n}^{2}$. Denoting nondimensional quantities with capitalized letter, $\Theta$ and $t^{*}$ :

$$
\begin{gathered}
X=\frac{x}{H} ; \quad Y=\frac{y}{H} ; \quad U=\frac{u}{u_{i n}} ; V=\frac{v}{u_{i n}} ; P=\frac{p}{\rho u_{i n}^{2}} ; \\
\Theta=\frac{T-T_{i n}}{T_{\mathrm{w}}-T_{i n}} ; \boldsymbol{t}^{*}=\frac{\boldsymbol{u}_{i n} . t}{\boldsymbol{w}_{\text {in }}}
\end{gathered}
$$

The governing equations of continuity, momentum, and thermal energy are then written in dimensionless form:

$$
\begin{gathered}
\frac{\partial U}{\partial X}+\frac{\partial V}{\partial Y}+\frac{\partial W}{\partial Z}=0 \\
\frac{\partial U}{\partial t^{*}}+\left(U \frac{\partial U}{\partial X}+V \frac{\partial U}{\partial Y}+W \frac{\partial U}{\partial Z}\right)=-\frac{\partial P}{\partial X}+ \\
+\frac{w_{i n}}{H} \frac{1}{\operatorname{Re}}\left(\frac{\partial^{2} U}{\partial X^{2}}+\frac{\partial^{2} U}{\partial Y^{2}}+\frac{\partial^{2} U}{\partial Z^{2}}\right) \\
\frac{\partial V}{\partial t^{*}}+\left(U \frac{\partial V}{\partial X}+V \frac{\partial V}{\partial Y}+W \frac{\partial V}{\partial Z}\right)=-\frac{\partial P}{\partial Y}+ \\
+\frac{w_{i n}}{H} \frac{1}{\operatorname{Re}}\left(\frac{\partial^{2} V}{\partial X^{2}}+\frac{\partial^{2} V}{\partial Y^{2}}+\frac{\partial^{2} V}{\partial Z^{2}}\right) \\
\frac{\partial W}{\partial t^{*}}+\left(U \frac{\partial W}{\partial X}+V \frac{\partial W}{\partial Y}+W \frac{\partial W}{\partial Z}\right)=-\frac{\partial P}{\partial Z}+ \\
+\frac{w_{i n}}{H} \frac{1}{\operatorname{Re}}\left(\frac{\partial^{2} W}{\partial X^{2}}+\frac{\partial^{2} W}{\partial Y^{2}}+\frac{\partial^{2} W}{\partial Z^{2}}\right) \\
\frac{\partial \Theta}{\partial t^{*}}+U \frac{\partial \Theta}{\partial X}+V \frac{\partial \Theta}{\partial Y}+W \frac{\partial \Theta}{\partial Z}=\frac{w_{i n}}{H} \times \\
\times \frac{1}{\operatorname{Pr} \operatorname{Re}}\left(\frac{\partial^{2} \Theta}{\partial X^{2}}+\frac{\partial^{2} \Theta}{\partial Y^{2}}+\frac{\partial^{2} \Theta}{\partial Z^{2}}\right)
\end{gathered}
$$

The Reynolds number is defined as $\operatorname{Re}=\frac{u_{i n} w_{i n}}{v}$, Prandtl number is $\operatorname{Pr}=\frac{v}{\alpha}$, and quantities $\alpha$ and $v$ are thermal diffusivity and kinematics viscosity of fluid, respectively. The Reynolds number signifies the ratio of inertia and viscous forces. The Prandtl number indicates the ratio of the momentum and thermal diffusivities. Denoting $W=\frac{\mathrm{w}_{\text {in }}}{H}$.

\section{Boundary CONDITIONS AND SOLUTION PROCEDURE}

The nondimensional form of the boundary conditions are: At the inlet port: $U=1 ; V=0 ; W=0 ; \Theta=0$

On the other solid walls: $U=V=W=0 ; \Theta=1$

On the adiabatic RACM walls: $U=V=0 ; \frac{\partial \Theta}{\partial X}=0$

The initial conditions for velocity and temperature fields are zero velocity and $\Theta=1$, respectively.

In order to investigate the effect of the inlet port's position, the value of $l$. A dimensionless variable for the positions of inlet port is defined as $L=\frac{l}{H}$. The velocity and temperature distributions were taken to be fully developed. From the preceding formulation, it shows that the nondimensional parameters governing this problem are $R e, P r, W, L$. In this study, the Prandtl number of the fluid is assumed to 0.7 . The Reynolds numbers also to fixed to $5.10^{3}$.

The tetrahedral hybrid $T$ grid type topological element was used for meshing the flow domain. 121852 nodes are used in the 3D model. The CFD code used in this study was a Fluent solver (Fluent, Inc. 2006) [14]. By using solution-adaptive refinement, such as gradient adaption of velocity, the model 
could be refined by adding cells to enable the independent velocity field to be better resolved and coarsen the rest of the model room. When adaption is used properly, the resulting mesh is optimal for the flow solution. In other words, computational resources are not wasted by the inclusion of unnecessary cells in the rest of model.
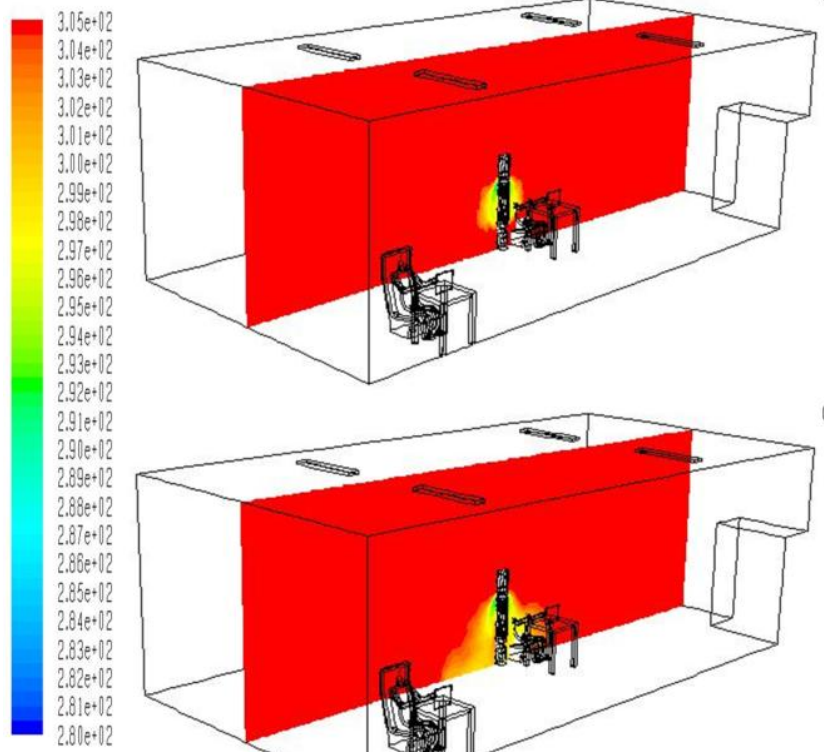

(a)

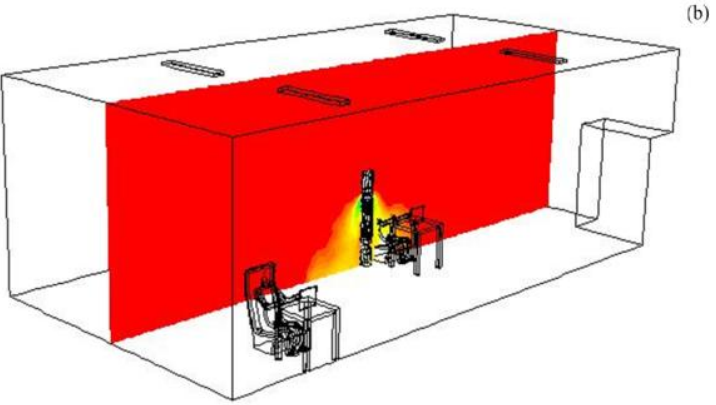

Fig. 3. Temperature distribution in the symmetrical plane: (a) $W=0.0117$ and (b) $W=0.0705$.

The fluid was assumed to be Newtonian, unsteady, and incompressible. A Boussinesq approximation was determined in order to consider the buoyancy effect. Computer code was solved, finite-difference and conservative equations were formed for the mass, momentum, and thermal energy. The solving method was couple implicit. The standard $k-\varepsilon$ turbulence model was selected, while the standard near-wall function was used in the near-wall treatment. In the solution control, a second order upwind method is specified. All the cases are iterated up to a convergence level of $10^{-6}$.

\section{VAlidation OF ACCURACY OF SimUlation}

Validation of the CFD using experimental work has been done by Huang et al. [11], [12]. Experimental and CFD data include the temperature and velocity in the occupied zone. The results of simulation are slightly different from the results of the experiments, around $11 \%$. The fairly good agreement between experimental and simulation results indicates that the CFD simulation is possible to use in the investigation of in-room characteristics of the RACM.

\section{EFFECT OF THE INLET PORT OPENING}

We analyzed the effect of the inlet port opening on airflow circulation cell by varying the height of the inlet port for a given RACM wall. A non-dimensional variable $(W)$, the ratio of inlet

port height to the height of RACM wall, is considered. It is necessary to develop a non-dimensionalized number for generalizing this analysis. The analyzed values of $W$ are provided in Table I. The minimum and maximum values of $W$ are 0.0117 and 0.0705 which correspond with inlet port heights of $0.02 \mathrm{~m}$ and $0.12 \mathrm{~m}$, respectively, for a RACM wall height of $2 \mathrm{~m}$. CFD simulation of airflow distributions were carried out in the modelled room after 500 seconds of cooling. The Fig. 3 depicts the temperature and velocity distribution in the $Y Z$ plane at the $X$ of $2.5 \mathrm{~m}$ from back room side(middle plane). When $W=0.0117$, the cool airflow circulation cell just concentrate in the occupied zone, most of the cool air from the inlet is sucked back to the outlet port by the negative outlet pressure to form a circulation cell. When $W=0.0705$, basically, the airflow circulation are still kept inside the cell, however, the airflow loss increases as the inlet port height is increased. Presumably, more of the cool air inlet flow reaches the room background and changes to a horizontal direction and moves further away from occupied zone and it may cause a larger temperature distribution in comparison with that of the smaller inlet port height cases. The vertical temperatures trends were recorded along $x_{1} x_{2}$, as shown in Fig. 4 for different heights of $0,0.1,0.3,0.5,0.7,0.9,1.1,1.2,1.3,1.5$, and $1.7 \mathrm{~m}$ from floor surface level. These heights correspond to Y of $0,0.058,0.176$, $0.294,0.411,0.529,0.647,0.764,0.882$, and 1 of the non-dimensional model. When $W=0.0117$, the temperature along $x_{1} x_{2}$ is almost constant up to 0.764 and higher than 0.764 , increased to $\Theta=1$. For the remaining $W$ values, temperature decreases only from floor surface level to 0.411 . The inlet port opening height affects the mass airflow rate entering the room. If the inlet port opening is smaller, the volume of air entering in the occupied zone is small and thus does not remove much heat from the occupied zone. Also, a small inlet port opening does not provide good regional air conditioning. In contrast, a large inlet port opening allows for a much volume of cool air to enter the room and provides better airflow circulation cell in the occupied zone. However, providing larger sized inlet port on the RACM will reduce the RACM wall' strength as well as consume more cool airflow, there is thus a trade-off among thermal comfort, strength of RACM system, anti-clogging system, and energy consumption issue. Fig. 4 also indicates that the occupied zone temperature is reduced by increasing the height of the inlet port opening. The air temperature zones are occupied at $P_{1}, P_{2}$, and $P_{3}$ are shown in Fig. 5.

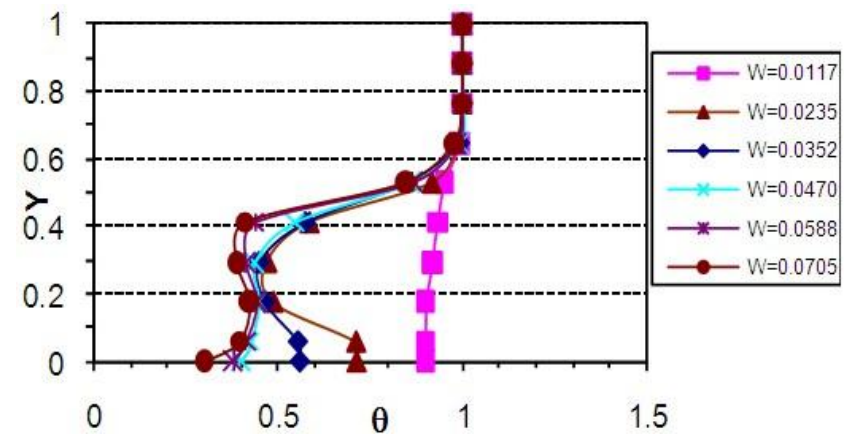

Fig. 4. Temperature distributions along middle of occupants for different W values.

When $W=0.0117$ only a small inlet port of area becomes the low temperature zone. When $W=0.0235$ to 0.0325 , the temperature change in the occupied zone is not significantly and the average temperature zone decreases linearly for 
planes $P_{1}$ and $P_{2}$. When $\mathrm{W}$ is higher than 0.0352 , the average temperature zone for planes $P_{1}$ and $P_{2}$ is around $\theta=0.6$. Judging from Fig. 5, the air temperature in plane $P_{3}$ is around $\theta=1$ as $W=0.0117$ to 0.0705 . Because the plane $P_{3}$ was far from the cool airflow source and cool airflow circulation cell located. The Fig. 5 show that, there is a gap between temperatures of the occupied zone with the RACM located $\left(P_{1}\right.$ and $\left.P_{2}\right)$ and the occupied zone without the RACM setup $\left(P_{3}\right)$. The low average air temperature is greatest at planes $P_{l}$ and $P_{2}$. This suggests that the indoor air temperature is lower at the area around the RACM located. Since the average low temperature for cases where $W=0.0352$ to 0.0705 has minimal variation, the case where $W=0.0352$ is identified as a suitable size for providing sufficient thermal comfort and consuming cool airflow, and strength of the RACM pipe.

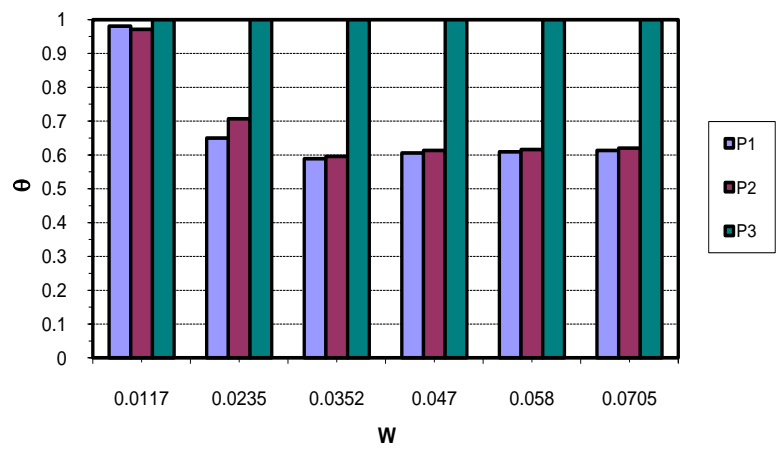

Fig. 5. Average temperature at $P_{1}, P_{2}$, and $P_{3}$ zones with different $W$ values

\section{EFFECT OF THE INLET PORT POSITION}

The effect of the inlet port position (i.e., the ratio of the inlet port position $(l)$ to the height of the RACM $(H)), L$, is varied for a given the RACM wall of $2 \mathrm{~m}$. The temperature distribution in the middle plane is shown in Fig. 6. For the smaller values $L$, the inlet port is near the outlet port and room surface. In this case, the cool airflow entering the room travels shorter distance before leaving the room. In addition, the cool airflow reaches the room surface quickly; then, the airflow circulation cell is less uniform and more airflow loss changes to a horizontal direction in the room. However, when $L$ is increased from 0.47 to 0.882 and the distance between inlet and outlet ports becomes longer, the cool airflow entering the room flows longer distance possible before reaching outlet port and room surface. A good airflow circulation cell will be filled almost the entire occupied zone with small loss that is located at the room surface. The reason for this is that, presumably, the distance between inlet and outlet port is long enough let the cool airflow be sucked back to outlet port. When $L=0.176$, the airflow circulation cell is less concentration in the occupied zone. This is attributable to the insufficient and non-uniform flow of circulation cell in the occupied zone. The vertical temperature trends were recorded along $x_{1} x_{2}$, as shown in Fig. 7 for different heights of $0,0.058,0.176,0.294,0.411,0.529$, $0.647,0.764,0.882$, and 1 from floor surface level. When $L=$ 0.176 and 0.294 , only area for height from $Y=0$ to 0.176 becomes the low temperature and after $Y=0.176$ where the temperature is held constant.

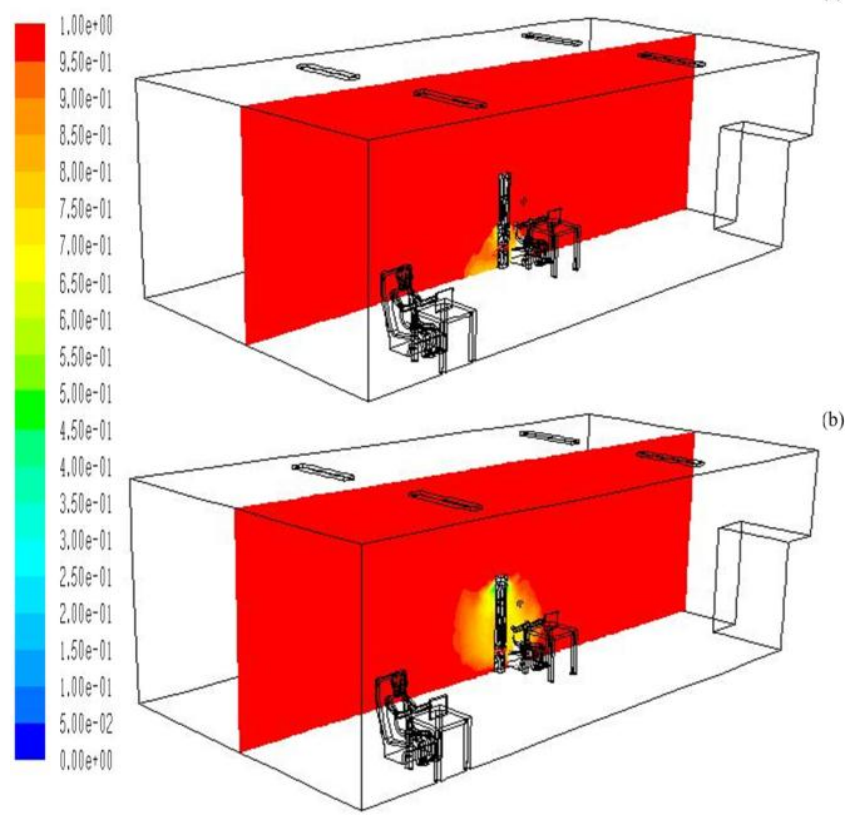

Fig. 6. Temperature distribution in the symmetrical plane: (a) $L=0.176$ and (b) $L=0.882$.

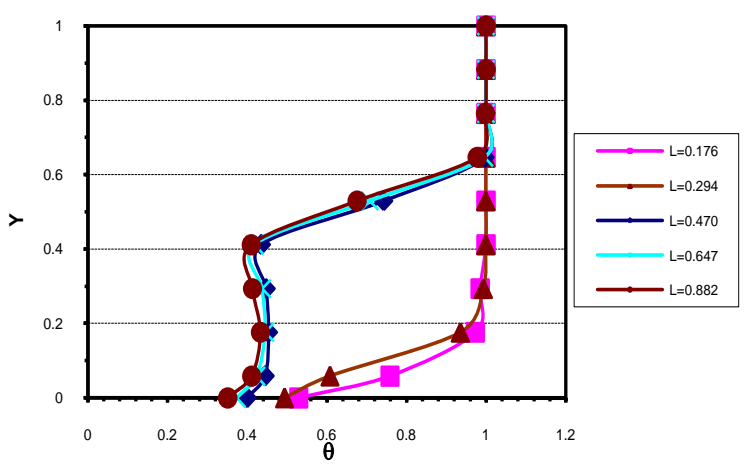

Fig. 7. Temperature distributions along middle of occupants for different $L$ values

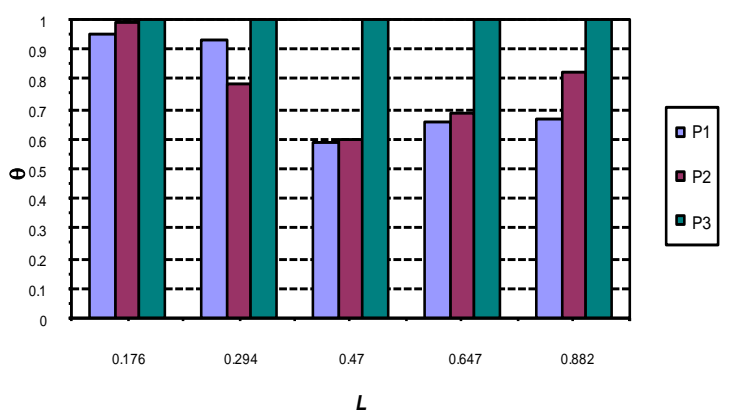

Fig. 8. Average temperature at $P_{1}, P_{2}$, and $P_{3}$ zones with different $L$ values

When $L=0.470$ to $0.882 \mathrm{~m}$ the temperature along $x_{1} x_{2}$ is below 0.74 up to a height of $Y=0.529$, above which it is constant. From theses trends it is clear that are with RACM system have lower temperature in the occupied zone. In all the vertical lines, the temperature is lowest when $L=0.882$ and increase until $L=0.176$. From this, it is seen that higher inlet port maybe better maintain the occupied zone's temperature at lower level that other locations in the room. However, when $L$ is higher than 0.470 , the temperature change is not significant and average temperature point where $L=0.470$ to 0.882 has minimal variation. The case where $L=0.470$ is identified as a 
suitable size for providing sufficient thermal comfort and anti-clogging system for the RACM. Fig. 8 shows the predicted area of the low temperature zone for planes $P_{1}, P_{2}$, and $P_{3}$. When $L=0.167$ to 0.47 , the average temperature zone decreases linearly for plane $P_{1}$ and $P_{2}$, whereas plane $P_{3}$ yields same average temperature of $\theta=1$ for all values of $L$. When $L$ is higher than 0.47 , the average temperature zone increases for plane $P_{1}$ and $P_{2}$, however, the temperature change is not significant and the average low temperature is around 0.6. All of these findings support proving the RACM that, under suitable adjustments of $L=0.47$, the highest level of thermal comfort demands and anti-clogging RACM system potential can be achieved.

\section{CONCLUSIONS}

The paper investigated the effect of non-dimensional variables such as the inlet port opening $(W)$ and inlet port position $(L)$ on airflow circulation cell establishing process to improve individual thermal comfort and energy efficiency in the RACM system. We varied the parameters $W$ and $L$ from 0.0117 to 0.0705 and 0.176 to 0.882 , respectively. Based on our results, $W=0.0352$ is the suitable inlet port opening for trade-off between thermal comfort and RACM strength, and energy-saving issue. Under the suitable adjustment of $L=$ 0.47 , the highest thermal comfort and anti-clogging system can be achieved. Therefore, these simulation results will be a valuable basis for the research and the design of the RACM system.

\section{ACKNOWLEDGMENT}

This work was supported by The National Foundation for Science and Technology Development, Vietnam.

\section{REFERENCES}

[1] Y. Murakami, M. Terano, K. Mizutani, "Field experiments on energy consumption and thermal comfort in the office environment cotrolled by occupants' requirements from PC terminal," Build. Environ, vol. 42 , pp.17-27, 2007.

[2] ISO 7730, Moderate thermal environments, "Determination of PMY and PPD indices and specification of the conditions for thermal comfort," International Organization for Standardization, Geneva, 1994.

[3] Q. Zeng, J. Kaczmarczyk, A. K. Melikov, and P. O. Fanger, "Perceived air quality and thermal sensation with personalized ventilation system," in Proc. of 8th Inter Conf. of Air Distribution in Rooms-Room Vent 2002, Copenhagen, Denmark, pp. 61-64, 2002.

[4] A. K. Melikov, "Personalized ventilation," Indoor Air, vol. 14, pp. 157-167, 2004.

[5] P. O. Fanger, "Indoor air quality in the 21st century: search for excellence" Indoor Air, vol. 10, pp. 68-73, 2000.
[6] J. Kaczmarczyk, A. K. Melikov, and P. O. Fanger, "Human response to personalized ventilation and mixing ventilation," Indoor Air, vol. 14, pp. 17-29, 2004.

[7] K. D. Huang, S. C. Tzeng, W. P. Ma, and M. F. Wu, "Intelligent solar-powered automobile-ventilation system," Applied Energy, vol. 80, pp. 141-154, 2005.

[8] K. D. Huang, S. C. Tzeng, T. M. Jeng, and W. D. Chiang, "Air conditioning system of an intelligent vehicle cabin," Applied Energy, vol. 83, pp. 545-557, 2006

[9] K. D. Huang, N. A. Tuan, and Y. C. Shih, "Energy-saving and thermal comfort studies of a regional air-conditioning mechanism" in Proc. of the IME, J Power Ener, 2010, vol. 223, pp. 12-33.

[10] K. D. Huang and N. A. Tuan, "Numerical analysis of an air-conditioning energy-saving mechanism," Buil. Simulo: Int. J. vol. 3 , pp. 63-73. 2010.

[11] K. D. Huang and N. A. Tuan, "Numerical and experimental analysis of an air conditioning energy-saving mechanism," presented at 2nd Asian Symposium on Computational Heat Transfer and Fluid Flow, Jeju, Korea, 2009.

[12] K. D. Huang and N. A. Tuan, "Low cooling energy consumption of room with regional air conditioning mechanism," Int Conf. Pow Eng-09 (ICOPE-09), Kobe, Japan, 2009.

[13] GAMBIT 2.4. User guide, FLUENT Inc., all volumes, Lebanon, $\mathrm{NH}$ 03766, USA, 2007.

[14] FLUENT 6.2.User guide, FLUENT Inc., all volumes, Lebanon, NH 03766, USA, 2005.

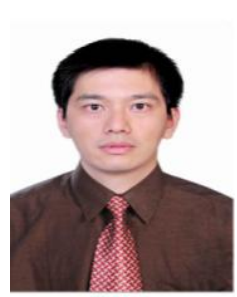

Nguyen Anh Tuan was born on Sep. 10, 1979 at Hanoi, Vietnam. He is studying Ph.D in Institute of Mechanical and Electrical Engineering, National Taipei University of Science and Technology, Taipei, Taiwan, R.O.C. He was with the department of Mechanical Engineering, HUST, Vietnam from 2002 to 2004. He was teaching Assistants and Research Assistants, Dayeh University (DYU), Changhwa Taiwan, R.O.C. He received M.Sc. in DYU during 2004 to 2006. He was teaching Assistants and Research Assistants, National Taipei University of Technology (NTUT), Taiwan, from 2006 to 2012. He received the Ph.D degree in NTUT. He worked in International R\&D Office, Takming University of Science and Technlogy, Taipei, Taiwan, 2011-2013. $\mathrm{He}$ is working in Dept. of International Co-operation, Ministry of Sci. and Tech., Hanoi, Vietnam, 2013.

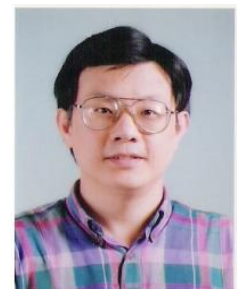

K. David Huang received Ph.D his degree from Mechanical Department University of Michigan Ann Arbor, USA. He is a professor and Chairman of Vehicle Department, National Taipei University of Technology, Taipei, Taiwan, R.O.C. $\mathrm{He}$ is specialized in Advanced Vehicular Power System, Metallic Fuel Cell, Regional Air Conditioning, and Cooling Engineering. He got his Bachelor Degree in Department of Mechanical Engineering, National Taiwan University, Vietnam during1978 to 1982 . He got his Master degree in Department of Mechanical Engineering, University of Michigan - Ann Arbor, USA during 1985 to 1987. He got his Ph.D Degree in Department of Mechanical Engineering, University of Michigan - Ann Arbor, USA, 1987 to 1992. He was one of the committee members in Cooling System Standards Committee, SAE, USA from 1991to1997.He was an Assistant Professor in Dayeh University, Taiwan from 1997 to 2002. He was associate Professor, Dayeh University, Taiwan from 2002 to 2006 . He is professor in National Taipei University of Technology, Taiwan now. 